\title{
Identités symbiotiques et dialogisme chez Herménégilde Chiasson et Andrée Lacelle
}

\author{
François PARÉ \\ Université de Waterloo
}

Si notre époque cherche encore souvent à repérer dans les habitus d'un sujet dit post-identitaire les cas de ruptures et les uniformités fictives - car seul le rompu au sein de son histoire personnelle garantirait sa vérité historique -, elle aspire aussi à éclairer la plénitude du sens offerte par les notions jumelles d'intermédiaire et de fragmentaire. Dans un bref essai sur Maurice Blanchot, Jérémie Leduc-Leblanc a évoqué toute la richesse de ce double mouvement: " [...] si le fragment met surtout en relief une certaine brisure entre l'être et le monde, il n'est pas la brisure, et s'il apparaît comme manque, il n'en demeure pas moins articulation» (Leduc-Leblanc: 62, nous 
soulignons). Il semble donc que la logique identitaire du sujet contemporain passe par la reconnaissance du fragment, comme si celui-ci formait le lieu d'une détermination particulière de ce sujet, alors que se déploierait, dans l'exiguïté de ce territoire élusif, une courte passerelle posée sur le vide qui l'avait toujours séparé des autres et le dénotait dans l'opacité de sa différence. Dans le cas du sujet minoritaire, qui nous intéressera ici particulièrement, la nécessité de ce paradoxe est encore plus grande, dans la mesure où elle imprime sur tous et chacun un déchirement de tous les instants entre la fidélité et le renoncement à la communauté d'origine.

Or la construction d'une subjectivité qui entretiendrait des relations plus harmonieuses avec le monde affleure dans un certain nombre d'œuvres littéraires du Canada français et d'Acadie où l'organicité positive du dialogue se présente comme une alternative à une confrontation dominants-dominés jugée stérile sur tous les plans. La recherche d'une voie médiane où se résoudraient certaines des tensions identitaires s'impose sans pour autant que soit mise en doute la spécificité historique du sujet minoritaire. Au contraire, cette histoire ne le force-t-elle pas à choisir, peut-être à cause du sentiment de fragilité qui l'habite, entre les termes d'une douloureuse contrariété trop souvent constitutive de sa différence?

Dès le tournant des années 1970, en Ontario français comme en Acadie, la littérature a eu pour mandat d'élucider ces tensions débilitantes. Pendant ces années charnières, une véritable anthropologie de l'identité a pu s'élaborer autour du théâtre et de la poésie. Certaines œuvres, cependant, par des auteurs comme Andrée Christensen, Andrée Lacelle, Hédi Bouraoui, Gérald Leblanc ou Herménégilde Chiasson, inspirées 
par le désir de rendre compte de la remarquable polyvalence des rapports symbiotiques entre le soi et l'autre, se sont élaborées en marge des débats sur l'identité. Plusieurs de ces textes aux fortes dimensions spiritualistes s'inspiraient d'esthétiques qui dépassaient largement les confins du Canada français et de l'Acadie, appartenant plutôt à des enjeux littéraires continentaux ou même mondiaux. Pour les fins de cette étude, deux dialogues poétiques, peu souvent commentés, alimenteront notre réflexion:Conversations, recueil de dialogues publié par Herménégilde Chiasson en 1998 et réédité en 2006 et Survenance, œuvre radiophonique à deux voix composée par Andrée Lacelle en 2000. Ces œuvres mettent en scène deux voix en alternance, mais elles ne reproduisent pas, en dépit du titre de l'ouvrage de Chiasson, les structures de la conversation. Bien qu'elles ne se répondent pas toujours, les voix se projettent plutôt comme des instances autonomes à l'affût de ce qui les unit et de ce qui les distingue. La forme dialoguée, en ce qu'elle favorise l'alternance de voix convergentes, s'éloigne aussi des tensions qui structurent notamment le théâtre, puisqu'aucun objectif de réalisme n'y préside. Ne renonçant pas à «prendre la parole», les voix en présence assurent pourtant la mise en forme d'un projet commun de renouvellement des espaces identitaires, assurant le passage intermittent du sens entre les interlocuteurs. Faisant appel au fragment et à l'inachèvement, le dialogue permet donc d'élaborer des subjectivités tant solitaires que traductives, à la confluence de leur solitude existentielle et d'un fort désir d'empathie et de collaboration.

Andrée Lacelle et Herménégilde Chiasson avaient, du reste, publié à la fin des années 1990, dans un numéro de la revue Francophonies d'Amérique, une brève correspondance qui 
portait, entre autres choses, sur le statut de l'écrivain et sur les formes que prenait la création dans leurs milieux artistiques respectifs de l'Ontario français et de l'Acadie. Tandis que Chiasson relate son découragement devant l'avenir problématique réservé aux Acadiens, Lacelle évoque pour sa part l'image d'une société franco-ontarienne active, symbiotique et fortement intégrée. Pourtant, dans ces lettres, le devoir d'identité, particulièrement ressenti dans les cultures minoritaires, semble oppressif et même fallacieux. Chiasson s'insurge contre l'« Acadie comme slogan, comme marque de fabrique ou comme attrait touristique » (p. 179). Du même souffle, l'écrivain peut-il souhaiter se départir entièrement de ses responsabilités devant les exigences de l'histoire acadienne? Andrée Lacelle réagit au pessimisme de son correspondant dans une lettre datée du 11 novembre 1997, dans laquelle elle propose plutôt la déconstruction systématique des conflits et des oppositions hérités du passé. Évoquant le travail du sculpteur franco-ontarien Roland Poulin qui réussit dans ses œuvres à « articuler le vide » à l'aide de fragments agglutinés, Lacelle dit rechercher, elle aussi, « l'union des forces contraires, la symbiose des polarités » (p. 185). À l'image même de l'échange épistolaire, l'écriture doit viser la construction d'une culture ligamentaire qui prendrait en charge de façon positive les douleurs du passé collectif. " De quoi rêvons-nous présentement collectivement parlant», se demande par ailleurs Chiasson, " et le fait de rêver en commun est-il une illusion qui devrait nous déserter considérant le fait que nous n'avons peutêtre pas eu de rêve autre qu'une révolte anarchiste appuyée in extremis sur un sentiment d'exclusion? Alors comment faire pour générer un rêve plus positif » (p. 171)? Cet espoir de créer par la littérature une subjectivité nouvelle et tournée vers 
l'avenir semble donc animer les deux écrivains à la fin des années 1990. Les œuvres dialogiques qu'ils publient tour à tour quelques mois plus tard constituent à n'en pas douter des tentatives de transcender les identités binaires et de "générer un rêve plus positif » à même l'épuisement de ces polarisations.

\section{Le sujet traductif}

Dans une note critique sur le théâtre québécois contemporain, Marie-Christine Lesage constate le décloisonnement récent des arts de la scène au Québec et ailleurs en Amérique et "la présence accrue d'œuvres interartistiques" où affleurent des formes connexes de théâtralité, s'apparentant au dialogue, à l'exposé et même à la sculpture: "Certaines interactions entre la danse et le théâtre, par exemple, ou encore entre le théâtre et les techniques de l'image tendent à faire émerger une théâtralité déplacée dans sa matière scénique, en retrait par rapport au symbolique et au sujet, esquissant de nouvelles façons de représenter l'imaginaire en scène» (Lesage, p. 177). Ces pratiques traductives entre les divers discours de la performance correspondent de façon générale aux parcours d' « interférence créative » que souligne Sherry Simon dans son livre Traverser Montréal. Une histoire culturelle par la traduction. En effet, les itinéraires inattendus et les croisements incessants de la vie moderne "vont à l'encontre du modèle binaire dominant des cultures majeures et mineures, du centre et de la périphérie, à l'encontre des relations hiérarchiques, et en faveur d'un terrain plus vaste d'interactions entre les minorités » (p. 279). Les déplacements discursifs et identitaires qui sont interpellés par le paysage urbain témoignent d'une 
recherche de lieux consensuels et d'échanges qui dépassent de beaucoup le contexte particulier de la ville et de ses itinérances. Surtout, ces théâtralités " douces », pour ainsi dire, permettent d'entrevoir l'émergence d'identités moins hiérarchiques qui remettent en cause la pertinence pour le sujet contemporain de la notion même d'altérité. Tout porte à penser que l'intermédialité, entendue comme médiation systématique de la différence, définit le mode d'être du sujet minoritaire contemporain.

Dans les lieux les plus fragiles de son appartenance, le sujet affiche ainsi sa tranquille complexité, car la conscience profonde de sa différence s'amortit toujours dans la plénitude de son abandon au sein des forces dominantes. Être soi n'est donc jamais s'appartenir en propre. Du moins, pas uniquement. Le sujet traductif s'exprime alors autant sur le mode de la résistance que sur celui du don et de l'accueil. Le travail de l'identité s'appuie sur le sens premier accordé au même, à l'identique. À quel moment le sujet s'efface-t-il, devient-il trace vivante de sa communauté affirmée? Alexis Nouss note, il est vrai, que l'alternance identitaire n'est jamais «contenue ou réduite au sein d'une harmonie ordonnée» (p. 105). Dans son face-à-face quotidien avec la diversité du monde, le sujet minoritaire ne fait qu'entrevoir sa propre altérité. Il est bien cette rupture, quel que soit son besoin de faire communauté. N'est-ce pas dans ces moments de doute qu'il aperçoit l'étendue de sa désaffiliation d'avec le monde? Car, étrange et étranger en lui-même, ce sujet métissé projette le sacrifice inévitable de sa propre reconnaissance. En lui, la marginalisation et l'itinérance se racontent en un récit tronqué dont il n'est pas sûr d'être l'auteur. Du même souffle, comme le fait voir avec force l'anthropologue Ronald Niezen au sujet des peuples 
autochtones, seul l'exercice du langage comporte des valeurs rédemptrices au-delà de tous les ostracismes et ouvre la voie à de nouvelles " épistémologies » de la rencontre (p. 185). C'est pourquoi, aux yeux de ce chercheur, la construction de l'identité ne se conçoit plus aujourd'hui que dans l'exercice complexe de la compassion et l'engagement de chacun envers la justice sociale. Toute distinction, qui serait réclamée par le sujet au nom de l'exclusion et du manque, doit entraîner, selon Niezen, le déploiement de théâtralités communes. Le défi des minorités, quelles qu'elles soient, réside aujourd'hui dans la ténuité de cet argument qui fonde pourtant le devenir des sociétés composites actuelles.

$\mathrm{Si}$ toutes ces approches cherchent à décrire des phénomènes de rencontre interculturelle et de créolisation, elles ne renoncent jamais à une dualité irréductible que semble nécessiter leur mise en langage. Traduire la différence, n’est-ce pas se l'approprier pour enfin la tenir à distance? La « poétique de la relation ", dont Édouard Glissant a décrit les bienfaits et même l'inévitabilité pour les sociétés colonisées, reste malgré tout une politique de l'intervalle, car, comme l'avoue Glissant lui-même, l'attachement au pays d'origine fait obstacle : « [l]e lieu d'où on émet la parole, d'où on émet le texte, ce lieu-là est immense » (p. 29). Dans une conférence ultérieure, Glissant précisera le fondement même de la «relation» dans la différence : «... s'il n’y a pas de différences, il n’y a pas de relation» (p. 97). Ainsi, le sujet contemporain, tel qu'il est souhaité, ne saurait être pure relation. Quelque chose d' « immense » l'entrave, sans doute un attachement au « lieu » de sa première manifestation, quelle que soit la douloureuse pauvreté de ce lieu fondamental. 
Or, dès la fin des années 1960 au Québec et à la fin des années 1990 au Canada français, certains textes, assez souvent en marge des discours et tendances nationalistes et identitaires, tentent de désamorcer de façon radicale les tensions dialectiques qui subsistent au sein de l'analyse postcoloniale du sujet contemporain et de ses identités. Ces mouvements, inspirés par les spiritualités amérindiennes et orientales, mettent en jeu non seulement les questions relatives au lieu de naissance et à l'appartenance identitaire, mais aussi les formes génériques qui permettent au sujet de se saisir dans un pur état de relation : " Il y a, dans cette intelligence de la signification », précise Denise Brassard dans son livre sur la poésie québécoise, « quelque chose d'essentiellement actif et ouvert, une tension, une réceptivité qui ne se laisse pas réduire à une perception ni à une 'conscience de'. Nous ne sommes pas ici dans le concept, mais dans l'intelligible, dansl'intelligence en mouvement» (p.347). Pour les besoins de cette étude, nous nous contenterons de suivre à la trace la quête d'une subjectivité minoritaire, non plus braquée sur le sens à donner à la différence, mais issue du rapprochement avec les autres et d'une culture de l'échange. Dans un entretien avec Paul Savoie, Andrée Lacelle souligne d'ailleurs la nécessité de conjuguer solidarité et solitude :

Où l'on peut voir des polarités dans les termes solitaire et solidaire, je perçois surtout une convergence. En effet, l'un ne dédit pas l'autre: l'être seul et l'être en collectivité, c'est la présence de bout en bout, et cet impératif de la présence, les poètes en sont des familiers, surtout en milieu minoritaire où présence éparpillée, précarité et durance sont soudées comme cœur et destin (Lacelle, dans Savoie, 175).

Ce très beau texte inscrit le dialogue au cœur même du sujet minoritaire, lui qui évolue toujours à la fois en lui-même et 
hors de lui-même : "Tout être est à la fois souffle et racines » (p. 175). Stéphane Vibert fait enfin remarquer, dans une étude sur le pluralisme des sociétés contemporaines, la dimension « volontaire et personnelle » de ce qu'il appelle « l'appartenance élective ». Tout en rejetant les " traditions hiérarchisantes », le sujet cherche à " conjuguer l'autonomie et l'intégration, la liberté et la solidarité ", afin de faire voir le paradoxe fondamental sur lequel il construit et justifie sa différence (Vibert, p. 39). Chez Andrée Lacelle comme chez Herménégilde Chiasson, les débats sur l'identité, surtout dans les sociétés marginalisées, affichent ainsi une forte préoccupation éthique.

\section{La terrible fatigue du temps}

L'œuvre d'Herménégilde Chiasson s'accompagne d'une présence auctoriale de tous les instants. Dans les textes poétiques comme dans les interventions publiques de l'auteur, chacun pressent le rôle déterminant de cette conscience énonciatrice qui, tout en cherchant à décrire la complexité et les contradictions propre à l'Acadie contemporaine et aux sociétés actuelles, préside, analyse, évalue, trie, condamne et synthétise. Dans un passage de sa correspondance avec Andrée Lacelle, Chiasson semble rejeter le personnage de l'intellectuel acadien auquel il est souvent identifié, mais il est clair que cette posture de l'analyste se trouve profondément reflétée dans l'ensemble de son œuvre.

Dans ce contexte, il faut voir la parution de Conversations en 1998 comme un événement assez particulier. En effet, ce recueil poétique, composé de 999 
répliques numérotées et attribuées à deux figures pronominales, Lui et Elle, tranche radicalement par la multiplicité apparente des points de vue exprimés et par la forme dialogique qui fractionne le texte en lui conférant l'allure d'une série d'aphorismes. Il ne s'agit d'ailleurs pas d'une pièce de théâtre (sa représentation sur scène paraît impensable, tant elle serait fastidieuse). Les structures syntaxiques nominales, les répétitions lexicales et l'absence de référentialité entraînent les «dialogues » vers l'énoncé poétique et la déclamation. Dans sa préface à l'édition de 2006, Pierre Nepveu souligne la pluralité des voix dans cette œuvre qui reste " une vaste scénographie à la fois de la conscience acadienne et, plus largement, de la subjectivité postmoderne » (p. 10). En ce qui nous concerne, la publication de Conversations correspond tout à fait à l'intérêt du poète, surtout à la fin des années 1990, pour le fragmentaire, le dialogique et le traductif. L'ouvrage met en présence deux voix parallèles qui, bien que différenciées sur le plan pronominal, restent presque toujours indistinctes. Au-delà de l'extrême fragmentation du texte, un effort de synthèse anime les deux personnages jusqu'à la dernière réplique, car le projet primordial de cette mise en scène consiste à redimensionner le sujet dans son appartenance à l'histoire et à le libérer de sa » prison intérieure » (\# 995, p. 153). En outre, sur le plan formel, la numérotation et l'interchangeabilité apparente des fragments jettent un doute sur la pertinence réelle des personnages masculins et féminins. Si les répliques attribuées à la femme sont l'expression d'une subjectivité marquée par le pronom je, ce n'est guère le cas de l'homme dont les interventions se font souvent à la troisième personne du singulier, ce qui confère à ses propos un détachement et une autorité plus grande. 
D’entrée de jeu, les deux personnages font état d'une grande fatigue existentielle. Il est clair que cet épuisement résulte de leur désenchantement devant l'histoire de faute et de mépris de soi qui est celle de leur culture d'origine. Ils se sentent seuls et abandonnés à eux-mêmes, ayant " perdu pour toujours quelqu'un qui nous tenait la main dans un monde sans merci » (\# 61, p. 14). L'amertume qu'ils ressentent envers cette origine chargée de crainte et de culpabilité semble motiver leur rencontre et leur désir de se confier l'un à l'autre. Leur amour se conjugue au rythme de cette répudiation répétée du passé collectif. Aux fragments 99 et 100, et encore dans de très nombreuses autres répliques, le texte de Chiasson fait clairement allusion à l'histoire difficile de l'Acadie dont le personnage masculin souhaite singulièrement se distancier. Ces répliques en écho sont cinglantes :

99. Lui Inutile de reformuler nos anciennes craintes, les anciens dieux de notre peur dorment désormais, défunts, silencieux de vigilance.

100. Lui Ne plus s'inquiéter de savoir si l'espace de notre provenance serait seul responsable de notre silence (p. 18).

Cette injonction confirme la valeur largement thérapeutique du dialogue, car la volonté de communiquer avec l'autre, de trouver dans cette altérité fraternelle les conditions propices à la transformation des anciennes identités, permet une première mise en récit d'une histoire personnelle affranchie des entraves historiques. À mi-parcours, au moment où les deux personnages se laissent aller de plus en plus à la confidence, la longueur des répliques passant de quatre à cinq ou six lignes, l'homme ne peut s'empêcher de revenir au passé : " Autrefois, on nous méprisait ouvertement et sans excuses allant même jusqu'à rire de notre manière de marcher en 
public» (\# 439, p. 60). En réponse, la femme exprime son espoir d'habiter un espace renouvelé par la symbiose des identités: " L'espace et tout ce qui s'ensuit, les raisons de l'habiter, de faire en sorte que les mots en deviennent une extension, une danse inoubliable, une déclaration d'amour » (\# 442, p. 60). Si le temps est chez Herménégilde Chiasson le signe d'une faille dramatique de l'histoire collective, l'espace devient en contrepartie la matière même d'une réinterprétation de l'historiographie. Or cette spatialité ne peut plus être liée aux affres de l'histoire acadienne, car elle forme justement la scène où cette histoire, désormais abstraite, ne peut plus avoir cours. Ce lieu intermédiaire est l'ultime symbiose, « le creuset même des grandes lignes de la présence » (\# 483, p. 67).

L'« obsédant projet » (\# 560, p. 79) de rédemption identitaire fait de Lui une figure christique (comme ailleurs sans doute dans l'œuvre de Chiasson) : « Dans la poussière de la nuit, il poursuivait sans relâche le travail de bossu qu'il avait entrepris dans la plus intarissable des convictions, son corps pliant sous le poids, montant sa croix dans les escaliers, se demandant les raisons d'une fin si abrupte » (\# 556. p. 78). Le livre lui-même dans le sacrifice de sa forme fragmentaire annonce à sa manière la fin de l'histoire communautaire et l'avènement d'un sujet électif. La réplique 606 marque l'apogée du cycle de destruction et de reconstruction mis en branle par le couple de devisants, couple inaugural et final à la fois. C'est Lui qui parle avec force: "Nous pénétrons ici le cœur du propos, celui qui laisse à penser que l'écriture est une sorte de vengeance, le livre étant écrit pour être mis en terre, pour qu'il en rejaillisse un arbre ou les fleurs tant attendues d'une colère inexprimable autrement » (\# 606, p. 87). Chez Herménégilde Chiasson, l'acceptation de l'origine, lieu mémoriel de douceur, 
de souffrance et de résignation, signifie également son dépassement. Car si le sujet résiste depuis toujours, sa résistance n'est pas tournée vers les autres dans leur différence, mais bien plutôt vers soi, car il est devenu avec le temps le signe de ce refus fondamental. Dans Conversations, le couple ne produit pas la synergie souhaitée. De ces 999 morceaux de sens, aucun récit ne semble émerger. Au contraire, le dialogue - s'il en est un - en fait voir les failles. Par ses structures nominales et ses phrases énumératives, souvent laissées en suspens, Conversations marque plutôt la fin du récit exemplaire de l'Acadie ou du moins de sa cohérence. Chez Chiasson, le langage permet tout de même de « remonter le cours de l'exil » (\# 925, p. 140) jusqu'au point de partage de la colère, là où le sujet, au bout de sa négativité, peut espérer franchir les frontières de sa dualité première.

\section{Ce lieu qui n'en est pas un}

Sans doute par leur double appartenance au livre publié et à l'oralité de la voix radiophonique, les dix-sept « conversations » de Survenance d'Andrée Lacelle cherchent, dès 2000, à rendre compte de personnages équitablement institués et moins polarisés. Si la poésie a pu représenter pour Lacelle l'expression d'une aventure individuelle, solitaire même, la structure dialogique de Survenance lui a permis d'introduire dans le déroulement même du texte et dans ses stratégies d'énonciation des impulsions en écho et des formes nouvelles de synergie. Un homme et une femme, s'étant aimés autrefois, se sont donné encore ici un "étrange rendez-vous » dans un lieu qui reste indéfini. Quelle est la nature de ce lien qui les 
rattache l'un à l'autre? Les souvenirs du bonheur passé suffisent-ils à expliquer leur pressant désir de se revoir? S'engage alors un échange ponctué d'interruptions et de silences, qui les conduira à comprendre le "décalage » qui les habite. Comme pour le couple mis en scène par Herménégilde Chiasson dans Conversations, c'est la disjonction, la faille au cœur de leur relation qui constitue la substance même de leur amour.

La situation dramatique de ces courts dialogues n'est pas sans ambiguïté, ni même sans causticité. Des moments d'impatience et des appels à l'action surgissent. Mais Survenance n'est pas exactement du théâtre. Les personnages en présence restent désincarnés et les tensions qui les habitent relèvent plutôt de l'inconscient. Or justement, l'œuvre radiophonique dépouille radicalement le tissu dramatique de sa corporéité; il ne reste de la présence scénique des acteurs que ces deux voix parallèles qui «surviennent» de façon imprévisible et passagère dans le vide ambiant. Quel visage accorder à ces simples pronoms LUI et ELLE qui portent la responsabilité de ce qui est dit et écrit ? ELLE est peut-être écrivaine et vit seule avec ses chats. LUI a un fort penchant pour la philosophie. C'est LUI qui soulève les grandes questions de l'existence. Ils sont d'accord sur presque tout. S'ils se sont un jour quittés, leur itinérance les a ramenés inévitablement au point de départ, car les affinités prévalent toujours sur les différences. Pourtant, tout départ comporte le risque de se perdre dans la distance : "ELLE. Toi et moi nous le savons: il n'y a pas de pays. Mais comment errer sans se disperser? » (Dialogue 13, p. 67) 
C'est par leur sentiment d'être tous deux étrangers à leur propre milieu, d'être là "par surcroît», qu'ils se rejoignent spirituellement. Cet homme et cette femme reproduisent assurément la binarité essentielle du couple, mais leur complémentarité semble nécessaire. En dépit de la fragilité de la rencontre, ces voix forment ensemble les vecteurs constitutifs du paradoxe de l'identité subjective, sans toutefois être des variantes d'un seul et même locuteur.

Les dialogues de Survenance sont marqués par l'intermittence. Les voix ne cessent de s'éteindre et ne reprennent jamais tout à fait au même endroit le fil de la discussion. Quelque chose semble s'être passé dans ces quelques répliques, mais nous n'en saurons rien de plus. Tout s'effacera aussitôt dans ces parcelles de vide qui, totalement dénuées de signes, servent pourtant de ligaments entre les dialogues. Les répliques des deux personnages ne débordent jamais le cadre qui leur est fixé par ces vastes zones de mutité. Ces temps d'arrêts, après quelques répliques seulement laissées en suspens, ne font que confirmer la pertinence initiatique des voix conjuguées. Jean-Pierre Martin souligne de belle façon l'émergence dans la littérature contemporaine d'un logos de la voix qui ferait contrepoids au «langage sacrifié » des sociétés de consommation:

Avant le langage sacrifié, ou plutôt, en-deçà, il y a un langage sorcier, le seul qui puisse produire une pensée qui ne soit pas préformée. Cet imaginaire de la vocalité intrinsèque défait le cloisonnement entre la pensée et l'émotion, la réflexion et le pathos, la spéculation et la sensation. Il reconnaît et revendique comme partie intégrante de la pensée son énonciation, son 'élan murmurant', son vocal spéculatif. (Martin : 15) 
Chez Andrée Lacelle, la publication de Survenance marque donc un intérêt renouvelé pour les vertus régénératrices de la vocalité. Reflétant un riche héritage philosophique et empruntant à la scène ses structures d'énonciation, le dialogue vocalisé acquiert ainsi une dimension spirituelle liée à l'origine de la parole. Il permet de détacher l'identité de ses dissonances matérielles et d'effacer les indices de l'appartenance.

Le personnage féminin mis en scène par Andrée Lacelle évoque à plusieurs reprises la profonde transformation qui l'anime et qui la laisse sans repères :

ELLE. .. . Quand saisie d'une sorte de fulgurance, j'ai contemplé mes limites, dans le même instant, je me suis sentie projetée hors du temps. Comme si je m'égarais dans un lieu qui n'est pas un lieu. Soudainement désorientée, sans le moindre repère, sans la perspective d'un retour à ce que j'avais été... (Dialogue 3, p. 26)

Plus tard, dans le fragment 10, la femme reprendra en des termes plus abstraits cette évacuation hors des balises du temps et de l'espace: "Hors de nous / Tout est trace » (p. 55). Chez Lacelle, la construction du sujet identitaire - surtout féminin -- ne s'effectue donc pas dans un contexte d'opposition entre le soi et les autres. Au contraire, le soi s'exprime par son empathie devant le drame de l'altérité pure. Pour y arriver, il doit s'abstraire de lui-même. Au dialogue 10, les figures de l'homme et de la femme, devenues «traces » et proprement «désorientées», parviennent à fusionner et cherchent ensemble le lieu où s'accomplirait une fois pour toutes leur rencontre: «Je cherche ce lieu comme on entend un appel », ditelle à la fin de ce bref échange. Et lui de répondre: «Eh bien, regarde-moi, là, où je t'appelle! » (Dialogue 10, p. 55). Le dialogue aura donc permis de faire apparaître 
l'intermédialité des figures de l'identité, d'abord traces, puis lieux d'accueil de la différence.

Le dialogue 11 revient d'ailleurs sur ce moment de traduction de soi. Cette fois, le personnage féminin, premier garant de la quête spirituelle, évoque un rêve d'enfermement inspiré par la lecture du prophète Ézéchiel. Dans ce rêve, dix portes fermées s'offrent de chaque côté d'une salle immense. Alors qu'une seule de ces issues est verrouillée, une voix se fait entendre à la femme agenouillée qui se laisse interpeller par l'interdit que représente le hors-champ de la porte fermée à clé. Doit-on ouvrir le battant vers l'intérieur ou vers l'extérieur? Cette question n'a de sens que dans une logique oppositionnelle. Dans Survenance, la rencontre n'est pas laissée aux fruits du hasard. Les lieux de l'identité sont électifs et l'enfermement une affaire de volonté. L'intertexte biblique, récit d'exil et d'identité compromise, constitue donc une véritable dénonciation des assises traditionnelles de l'identité. Renonçant aux limites de son origine, le personnage s'ouvre à la pensée dialogique et se doit de prendre le risque de sortir de lui-même pour " ouvrir grand les bras à ce qui est précaire » (Dialogue 16, p. 79). Les deux devisants concourent tout à fait sur la nécessité de dépasser les conventions de l'identité subjective, alors que la rencontre de leurs voix, même dans les nombreux moments de tensions, permet d'entrevoir un resourcement de l'identité. « Toujours j'attends cette sortie de moi-même qui me mettra au monde ", s'était exclamé un peu plus tôt le personnage masculin. Et ELLE avait répondu du même souffle par la nécessité du double :

Il dit : l'écho est sans cœur.

Je dis : l'écho est sacré (Dialogue 15, p. 76). 
Par ce qu'elle annonce sans pourtant en révéler la substance, la porte interdite est celle qui détient la clé d'une identité "vivante », libérée de son obsession pour l'exclusivité. Cette porte bat à la fois sur l'intérieur et l'extérieur.

Les dernières pages de Survenance font d'ailleurs l'éloge de la conversion en tant que forme révélée de la répétition. L'autre est donc de tout temps mon semblable. Le dialogue 16 évoque pour la première fois le couple inaugural emprunté à l'Ancien Testament. Mais Adam et Ève seront consignés au récit ambigu de leur filiation. Survenance se clôt sur la métaphore obsédante du visage, faisant écho peut-être à une lecture d'Emmanuel Levinas. Fantasmagorie de la mort, le dialogue convoque le sujet à sa disparition répétée et à sa résurgence éventuelle dans l'événement de la rencontre croisée. Chaque réplique, chaque fragment dialogique l'invite à s'ouvrir au regard renversé de la différence et d'y chercher le lieu de son recommencement.

\section{Réaménager la mémoire}

Dans ces deux œuvres exemplaires sur plus d'un plan, l'écriture dialogique permet de scénariser les modes traductifs d'une subjectivité contemporaine qui cherche ici à s'affranchir du travail de la mémoire collective. L'exercice met en présence deux voix parallèles dont la symbiose difficile, mais nécessaire, annonce la construction d'espaces de médiation plus ambigus, plus riches de possibilités et de retournements, plus résolument volontaires. Nombreux sont ceux au Canada français et en Acadie qui, à la fin des années 1990, considèrent que les grands récits mémoriels ne sont plus porteurs de cohésion identitaire 
et d'avenir collectif. En parlant de la déportation des Acadiens, Chedly Belkhodja invoque la nécessité d'effectuer un « réaménagement de la mémoire » qui ferait place à un débat critique et une " recherche de l'authenticité » (p. 221-222). Il est clair qu'un même enjeu force à réorienter la littérature, en Acadie comme dans l'ensemble du Canada français, vers la recherche de la polyvalence formelle et d'une plus grande ambiguïté des repères identitaires. Le recours à une esthétique du fragment et à des théâtralités plus souples et plus abstraites marque une volonté très claire, chez des écrivains comme Herménégilde Chiasson, Andrée Lacelle et plusieurs autres, de désamorcer la pesanteur du passé collectif, sans pour autant en nier totalement la pertinence.

\section{Bibliographie}

BelkhodjA, Chedly (2005), «L'Acadie. Une mémoire réaménagée de la reconnaissance au recommencement", dans Micheline Labelle, Rachad Antonius et Georges Leroux (dir.), Le Devoir de mémoire et les politiques du pardon, Sainte-Foy, Presses de l'Université du Québec, p. 211-228.

BRASSARD, Denise (2007), Le Soufflé du passage. Poésie et essai chez Fernand Ouellette, Montréal, VLB éditeur.

Chiasson, Herménégilde (2006 [1998]), Conversations, 2e éd., Sudbury, Prise de Parole. [Édition originale: Moncton, Éditions d'Acadie]

GLISSANT, Édouard (1996), Introduction à une Poétique du Divers, Paris, Gallimard.

LACELLE, Andrée (2001), Survenance, frontispice de MarieJeanne Musiol, Ottawa, Vermillon. 
LACELLE, Andrée et Herménégilde CHIASSON (1998), « Portraits d'auteurs. Andrée Lacelle de l'Ontario et Herménégilde Chiasson de l'Acadie ", Francophonies d'Amérique, no 8, p. 168-187.

LEDUC-LEBLANC, Jérémie (2007), «Le fragment ou la parole aveugle », dans Janine Gallant, Hélène Destrempes et Jean Morency (dir.), L'OEuvre littéraire et ses inachèvements, Longueuil, Groupéditions, p. 59-66.

LESAGE, Marie-Christine (2008), "Scène contemporaine et recherches interdisciplinaires au Québec », Globe. Revue internationale d'études québécoises, vol. 11, n², p.169-184.

MARTIN, Jean-Pierre (2003), « La critique et la voix : la double injonction », Études françaises, vol. 39, no1, p.13-23.

NEPVEU, Pierre (2006), «Préface» de Conversations d'Herménégilde Chiasson, Sudbury, Prise de Parole.

NIEZEN, Ronald (2009), The Rediscovered Self: Indigenous Identity and Cultural Justice, Montréal/Kingston, McGillQueen's University Press.

Nouss, Alexis (2002), "Métissage, transculture et singularité », dans Pierre Ouellet (dir.), Politique de la parole. Singularité et communauté, Montréal, Trait d'union, coll. «Le soi et l'autre », p. 99-112.

SAVoIE, Paul (2006), "Entretien avec Andrée Lacelle», dans Actes de création. Entretiens, Ottawa, L'Interligne, p. 167-176.

Simon,Sherry (2009), Traverser Montréal. Une histoire culturelle par la traduction, Pierrot Lambert (trad.), Montréal, Fides.

VIBERT, Stéphane (2007), «Le pluralisme des identités : mythe ou réalité? », dans Jacques Beauchemin et Mathieu Bock-Côté (dir.), La Cité identitaire, Montréal, Athéna, p. 31-42. 


\section{Résumé}

Dès le tournant des années 1970, en Ontario français comme en Acadie, une véritable anthropologie du sujet minoritaire s'est formée autour du théâtre de Jean Marc Dalpé et Michel Ouellette et surtout de la poésie minimaliste de Patrice Desbiens. D'autres œuvres, cependant, par des auteurs comme Andrée Christensen, Andrée Lacelle, Hédi Bouraoui, Gérald Leblanc ou Herménégilde Chiasson, inspirés par le désir de rendre compte de la remarquable polyvalence des rapports de symbiose entre le soi et l'autre, se sont élaborées en marge des débats sur l'identité. Plusieurs de ces textes aux fortes dimensions spiritualistes se sont inspirés d'esthétiques qui dépassaient largement les confins du Canada français et de l'Acadie, appartenant plutôt à des enjeux littéraires continentaux ou même mondiaux. Pour les fins de cette étude, deux dialogues poétiques, peu souvent commentés, alimenteront notre réflexion:Conversations, recueil de dialogues publié par Herménégilde Chiasson en 1998 et réédité en 2006, et Survenance, œuvre radiophonique à deux voix composée par Andrée Lacelle en 2000. À l'encontre du théâtre, le dialogue poétique a permis de présenter une vision plus organique de l'identité du sujet contemporain. 


\begin{abstract}
The early 1970s saw the birth in French-speaking Ontario and Acadia of the full anthropological figure of the minority subject, as reflected in a body of theatrical works by Jean Marc Dalpé and Michel Ouellette, as well as in Patrice Desbiens's minimalist poetry. However, other writers such as Andrée Christensen, Andrée Lacelle, Hédi Bouraoui, Gérald Leblanc, and Herménégilde Chiasson appeared instead to favour a more clearly symbiotic conception of the relations between the self and the others. Borrowing from spiritualistic traditions, their writings tended to reflect artistic movements at play not only in French Canada and Acadia, but also in North America and indeed the world. This essay explores in detail two lesserknown poetic dialogues, looking first at Conversations, published by Herménégilde Chiasson in 1998 (2006 for the paperback edition), and secondly at Survenance, a radio play written by Andrée Lacelle in 2000. Moving away from the centrality of the theatre, these works sought to present a more dialogic and inclusive vision of contemporary subjective identities.
\end{abstract}

